\title{
Pattern of referrals by Optometrists in Nigeria in relation to glaucoma and diabetic retinopathy
}

\author{
G.O. Ovenseri-Ogbomo ${ }^{1,2}$, O. Amiebenomo ${ }^{1}$ and A.E. Obahiagbon ${ }^{1}$ \\ ${ }^{1}$ Department of Optometry, University of Benin, Benin City, Nigeria, ${ }^{2}$ Department of Optometry, University of Cape Coast, Cape Coast, \\ Ghana
}

Referrals from primary care practitioners such as optometrists to other health care providers is critical to the health care delivery system of any society. This study explores the pattern of referrals of Nigerian optometrists with respect to glaucoma and diabetic retinopathy. A cross-sectional survey using a 28 -item questionnaire was administered to optometrists attending the $37^{\text {th }}$ annual general meeting of the Nigerian Optometric Association. Of the 58 respondents, $51(87.9 \%)$ of the respondents based their diagnosis of glaucoma on the triad of raised intra-ocular pressure (IOP), cup-disc (C/D) ratio and characteristic visual field defect while the remaining seven (12.1\%) based their diagnosis of glaucoma on raised IOP and C/D ratio. Fiftyfour $(93.1 \%)$ requested for a fasting blood glucose test (FBG) from patients who presented with ocular signs indicative of diabetes or diabetic retinopathy. When diabetic retinopathy was observed in a patient, 16 $(27.6 \%)$ responded to refer the patient to an ophthalmologist while eight $(13.8 \%)$ agree to refer the patient to a medical personnel/physician. Forty-four $(75.9 \%)$ of the respondents reportedly performs visual field test (VFT) while $14(24.1 \%)$ do not perform VFT. A total of $31(53.4 \%)$ of the respondents reported that they will initiate treatment for a glaucoma patient before referring the patient to an ophthalmologist while $10(17.2 \%)$ will refer glaucoma patient outright to an ophthalmologist. With respect to the stage of diabetic retinopathy that will warrant a referral, $42(72.4 \%)$ of the respondents refer the patient at any stage when the diagnosis is made while $5(8.6 \%)$ will only refer the patient if there is no improvement in the condition presumably after follow-up care. More female respondents were likely to refer glaucoma patients $(\mathrm{OR}=1.556$, $95 \% \mathrm{CI}=0.488-4.963)$ and diabetic retinopathy $(\mathrm{OR}=3.682,95 \% \mathrm{CI}=0.357-37.922)$ to ophthalmologists. Majority of Nigerian optometrists refer glaucoma and diabetic retinopathy patients to ophthalmologists; though more than half of the respondents will initiate treatment for glaucoma before referral.

Journal of Medical and Biomedical Sciences (2016) 5(3), 36-44

Keywords: Optometry, Ophthalmology, referral, Nigeria, glaucoma

\section{INTRODUCTION}

The World Council of Optometrists (WCO) states that "Optometry is a healthcare profession that is autonomous, educated and regulated (licensed/ registered) and optometrists are the primary healthcare practitioners of the eye and visual system, who provide comprehensive eye and vision care, which includes refraction and dispensing, detection/ diagnosis and management of disease in the eye and the rehabilitation of conditions of the visual system" (WCO, 2014). Optometrists are primary eye

Correspondence: Dr G O Ovenseri-Ogbomo, Department of Optometry, University of Benin, Benin City, Nigeria

Email: ovenseriogbomo@yahoo.com care professionals whose scope of practice in Nigeria is defined by the Optometrists and Dispensing Opticians Decree No 34 of 1989 (now Act Cap 09, Laws of the Federation of Nigeria 2004). Inherent in this Act is that in the day to day practice of optometrists, there are certain ocular conditions that are beyond the scope and competence of the optometrist and must therefore be referred to the ophthalmologists (who are medically qualified eye care physicians and surgeons) for further management. The practice of a referral system is to all intents and purposes in the patients' interest. It is to ensure that the patient is given the best of care available. 


\section{Referral pattern of Optometrists in Nigeria \\ Ovenseri-Ogbomo et al.,}

There have been instances where optometrists have been criticized for practicing beyond the scope of the practice and competence in Nigeria. In advanced countries there are stipulated pathways for referral (Harrison et al., 1988; Bowling et al., 2005). In Nigeria, no such clinical referral pathway exists. In the absence of clear cut referral pathways, referral practices are determined by individual primary care providers based largely on perceived competence rather than scope of practice. There is a paucity of studies exploring the referral interaction between optometry and ophthalmology in Nigeria. We conducted a preliminary survey to investigate the pattern of referrals by Optometrists in Nigeria with respect to glaucoma and diabetic retinopathy.

\section{MATERIALS AND METHODS}

\section{Study design}

A cross-sectional survey using a structured questionnaire to elicit responses from optometrists attending the 37th Nigerian Optometry Association (NOA) Conference and Annual General Meeting (AGM) in Umiahia, Abia State in July 2013.

\section{Data collection}

The 28-item questionnaire was divided into four sections. Section A asked questions relating to the respondents' demographic and practice history; sec- tions $\mathrm{B}-\mathrm{D}$ asked questions relating to referral practice with respect to glaucoma, diabetic retinopathy and low vision. A total of 100 questionnaires were randomly distributed to attendees at the NOA conference/AGM. The questionnaires were filled and returned within 30 minutes.

\section{Statistical analysis}

Responses were coded and analyzed using the statistical package for social sciences (SPSS) version 20.0. Appropriate descriptive and inferential statistics were performed. Binary logistic regression analysis was performed to predict referral patterns of optometrists. Results are presented in tables and appropriate charts.

\section{RESULTS}

Out of a total of 100 questionnaires administered, 61 were returned. Of these, three were incompletely filled given a response rate of $58 \%$. Thus analysis was based on the 58 questionnaires that were completed. The participants comprised 28 (48.3\%) males and $30(51.7 \%)$ females. The mean age of the respondents was $35.71 \pm 7.85$ years. The male respondents were significantly older than the female respondents $(p=0.043)$. Seventeen $(30.4 \%)$ respondents were in hospital practice $15(26.8 \%)$ were employed in an ophthalmologists' private

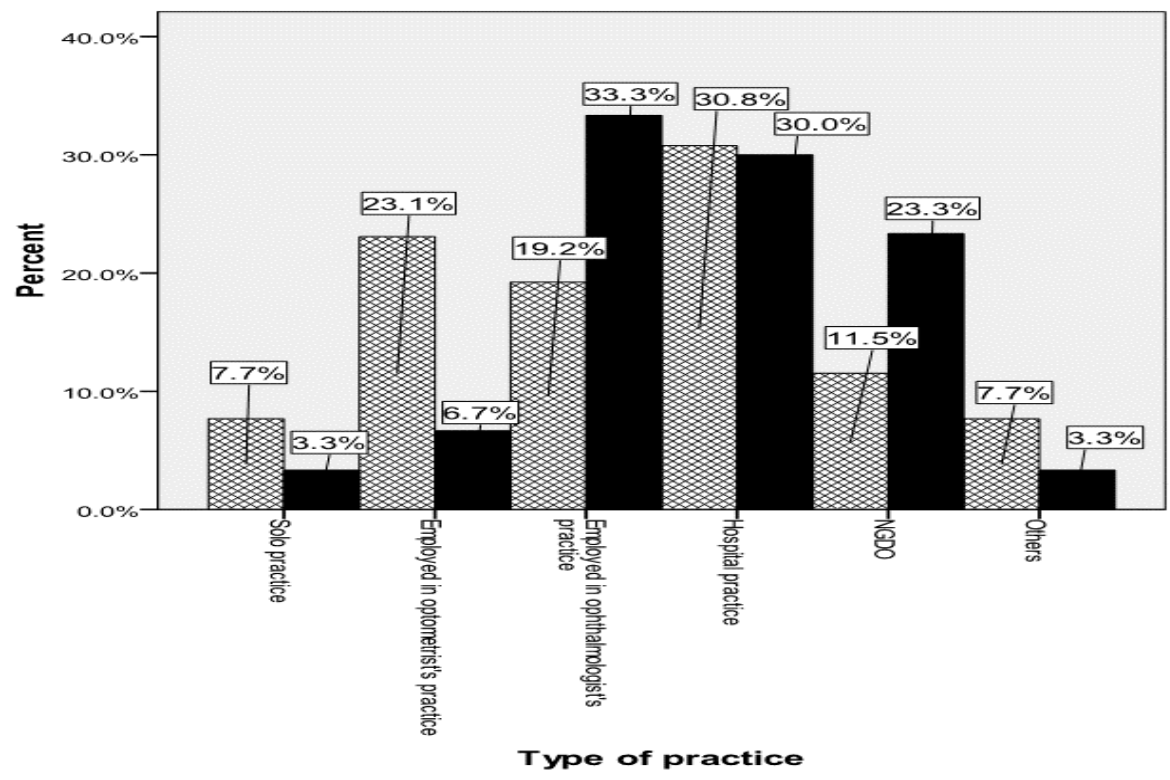

Gender Male

Figure 1: Mode of practice of respondents 


\section{Referral pattern of Optometrists in Nigeria Ovenseri-Ogbomo et al.,}

practice (Figure 1) whiles two respondents did not indicate their type of practice.

More females were employed in ophthalmologists' practice, hospital practice and in Non-governmental development organization (NGDO) while more males were in solo practice and employed in optometrists' practice. Nine $(15.5 \%)$ who reported their practice location practice in Rivers State while 6 $(10.3 \%)$ practice in Abia State. Other States had fewer respondents (Figure 2). Five of the six geo- political zones were represented albeit disproportionately. Only the North-East zone was not represented in the survey.

Thirty-two $(55.2 \%)$ of the total respondents have spent less than five years in practice (Table 1). The mean year in practice of respondents, post-National Youth Service Corp was $7.26 \pm 8.02$ years with a median of 3.50 years. The range was less than one year to 30 years.

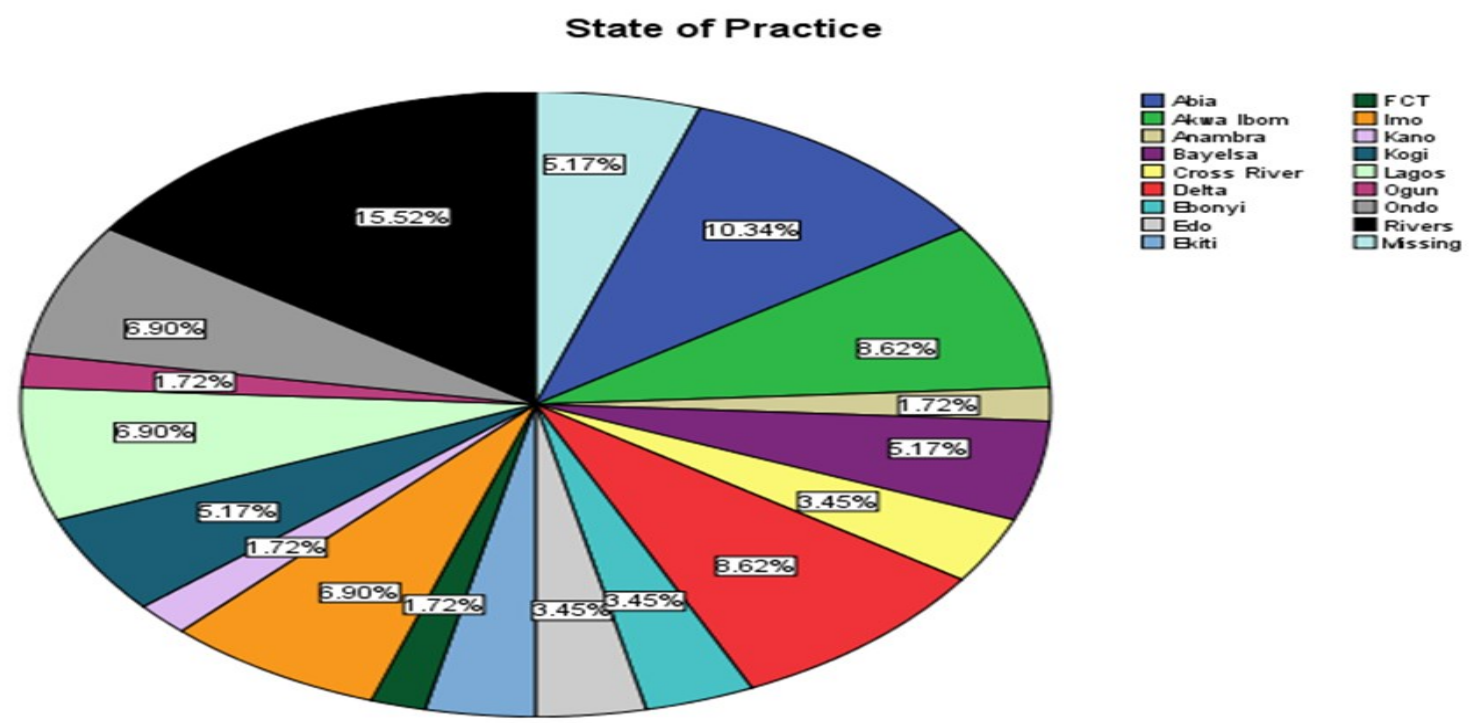

Figure 2: Practice location of respondents

Table 1: Distribution of number of years in practice of respondents

\begin{tabular}{lccc}
\hline & \multicolumn{3}{c}{ Gender } \\
\cline { 2 - 4 } Years in practice & Male (\%) & Female (\%) & Total (\%) \\
\hline $0.0-4.9$ & $13(46.4)$ & $19(63.3)$ & $32(55.2)$ \\
$5.0-9.9$ & $1(3.6)$ & $5(16.7)$ & $6(10.3)$ \\
$10.0-14.9$ & $7(25.0)$ & $3(10.0)$ & $10(17.2)$ \\
$15.0-19.9$ & $2(7.1)$ & $1(3.3)$ & $3(5.2)$ \\
$20.0-24.9$ & $3(10.7)$ & $1(3.3)$ & $4(6.9)$ \\
$25.0-29.9$ & $1(3.6)$ & $1(3.3)$ & $2(3.4)$ \\
$30.0-34.9$ & $1(3.6)$ & $0(0.0)$ & $1(1.7)$ \\
\hline
\end{tabular}

Data is presented as number and percentage 


\section{Referral pattern of Optometrists in Nigeria \\ Ovenseri-Ogbomo et al.,}

\section{Referral and practice pattern for glaucoma}

Fifty-one $(87.9 \%)$ of the respondents based their diagnosis of glaucoma on the triad of raised intraocular pressure (IOP), cup-disc (C/D) ratio and characteristic visual field defect while the remaining seven $(12.1 \%)$ based their diagnosis of glaucoma on raised IOP and C/D ratio. All respondents measured IOP in their practices. Twenty-six (44.8\%) re- not perform VFT. Of those who perform VFT, 26 $(59.1 \%)$ do so in their practices while $17(38.6 \%)$ refer patient to go for a VFT. One $(2.3 \%)$ respondent reports performing the VFT in the practice or requesting the test from outside the practice.

A total of $31(53.4 \%)$ of the respondents reported that they will initiate treatment for a glaucoma pa-

Table 2: Types of tonometers used by respondents

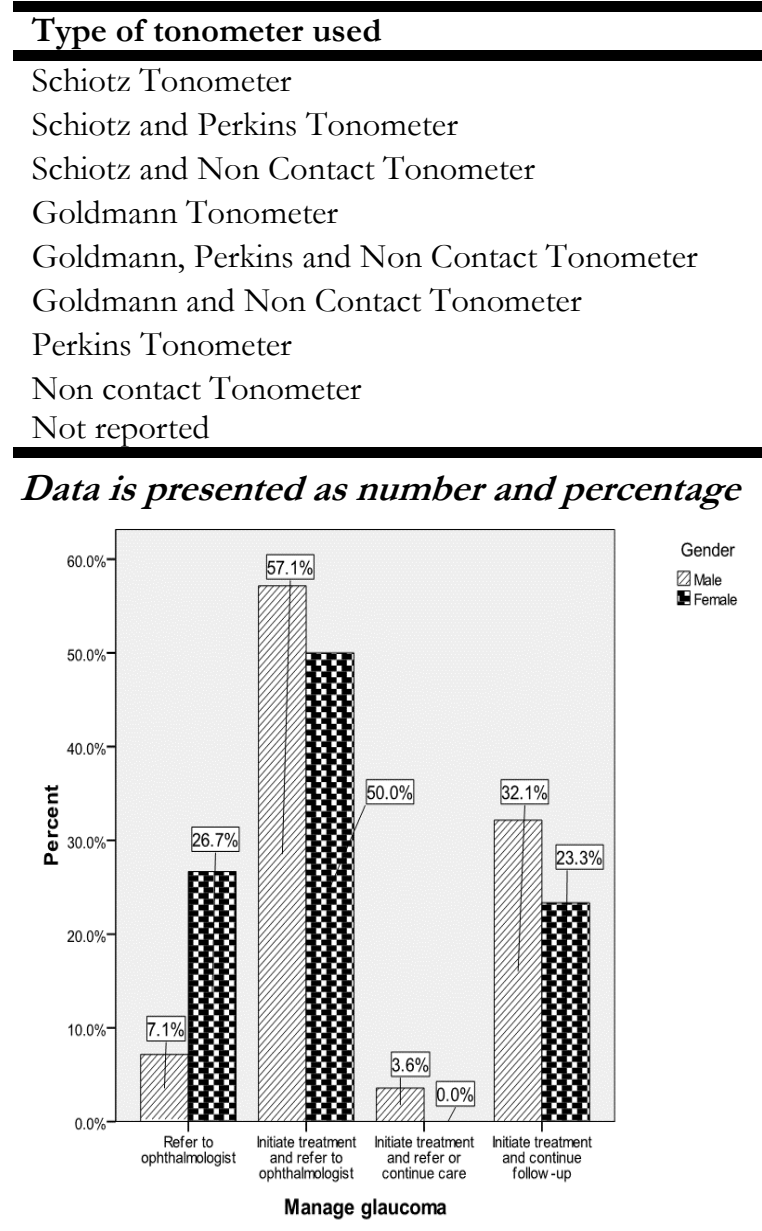

Figure 3: Action taken by respondents when a diagnosis of glaucoma is made

portedly use Schiotz indentation tonometer (Table 2).

Forty-four $(75.9 \%)$ of the respondents reportedly performs visual field test (VFT) while $14(24.1 \%)$ do

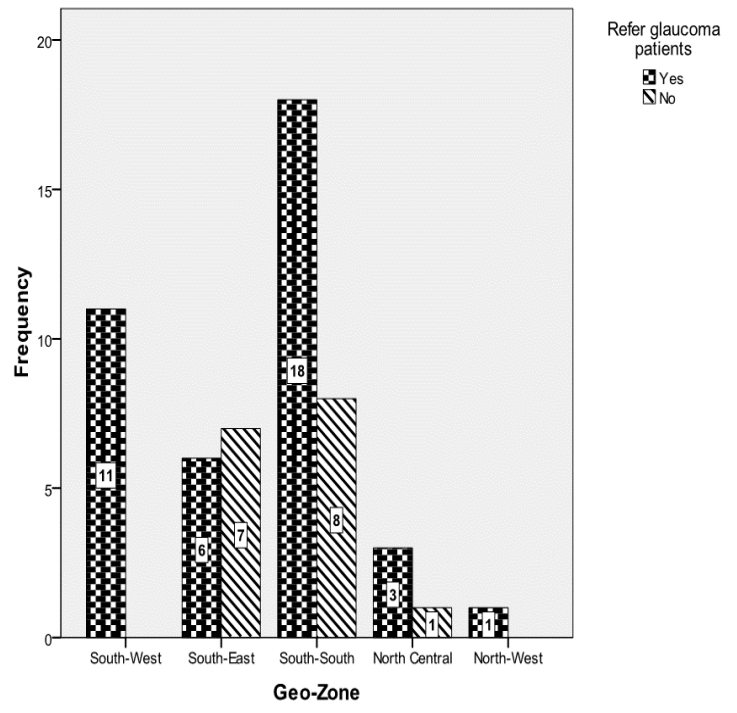

Figure 4: Referral pattern of glaucoma patients by geo-political zone

tient before referring the patient to an ophthalmologist while another $10(17.2 \%)$ will refer glaucoma patient outright to an ophthalmologist. Also 16 $(27.6 \%)$ of the respondents will initiate treatment and continue follow-up care for glaucoma patients. 
Only one $(1.7 \%)$ respondent will initiate treatment and refer to an ophthalmologist or continue followup care (Figure 3). More female respondents were likely to refer glaucoma patients to ophthalmologists than male respondents $(\mathrm{OR}=1.556,95 \% \mathrm{CI}=$ $0.488-4.963)$ although gender was not associated with referral pattern of respondents $(p=0.186)$. A logistic regression analysis to determine if gender, institution where optometric training was obtained or whether the respondents performed VFT or not influence the referral of glaucoma patients showed that none of these variables were significantly associated with referral behavior of participants with respect to glaucoma patients ( $p=0.696$ ). After controlling for gender, optometrists trained in University of Benin were more likely to refer glaucoma patients than those trained in Abia State University $(\mathrm{OR}=2.125,95 \% \mathrm{CI}=0.575-7.858)$ while those who perform VFT are less likely to refer glaucoma patients than those who do not perform VFT test $(\mathrm{OR}=0.738,95 \% \mathrm{CI}=0.175-3.124)$ (Figure 3$).$

On the criteria for referring a glaucoma patient, 22 $(44.9 \%)$ of those who responded to this question use large C/D ratio and raised IOP while another 21 $(42.9 \%)$ use raised IOP and VFT results. Two $(4.1 \%)$ respondents use IOP $>25 \mathrm{mmHg}$ while another four $(8.1 \%)$ use varying combination of raised IOP, VFT results and large C/D ratio as criteria for referring glaucoma patients. Whereas all $11(100 \%)$ respondents from the South-West zone will refer glaucoma patients, six $(46.2 \%)$ of 13 respondents from the South-East and 18 (69.2\%) of 26 respondents from the South-South zone will refer glaucoma patients (Figure 4).

\section{Referral pattern for diabetic retinopathy}

Of the 58 respondents, $54(93.1 \%)$ requested for a fasting blood glucose test (FBG) from patients who presented with ocular signs indicative of diabetes or diabetic retinopathy while four $(6.9 \%)$ did not order for test. Number of years in practice $(p=0.784)$, gender $(p=0.334)$ and institution where a respondent was trained (0.707) did not influence ordering FBST. When diabetic retinopathy was observed in a patient, $16(27.6 \%)$ of the respondents will refer the patient to an ophthalmologist while eight (13.8\%)

\section{Referral pattern of Optometrists in Nigeria} Ovenseri-Ogbomo et al.,

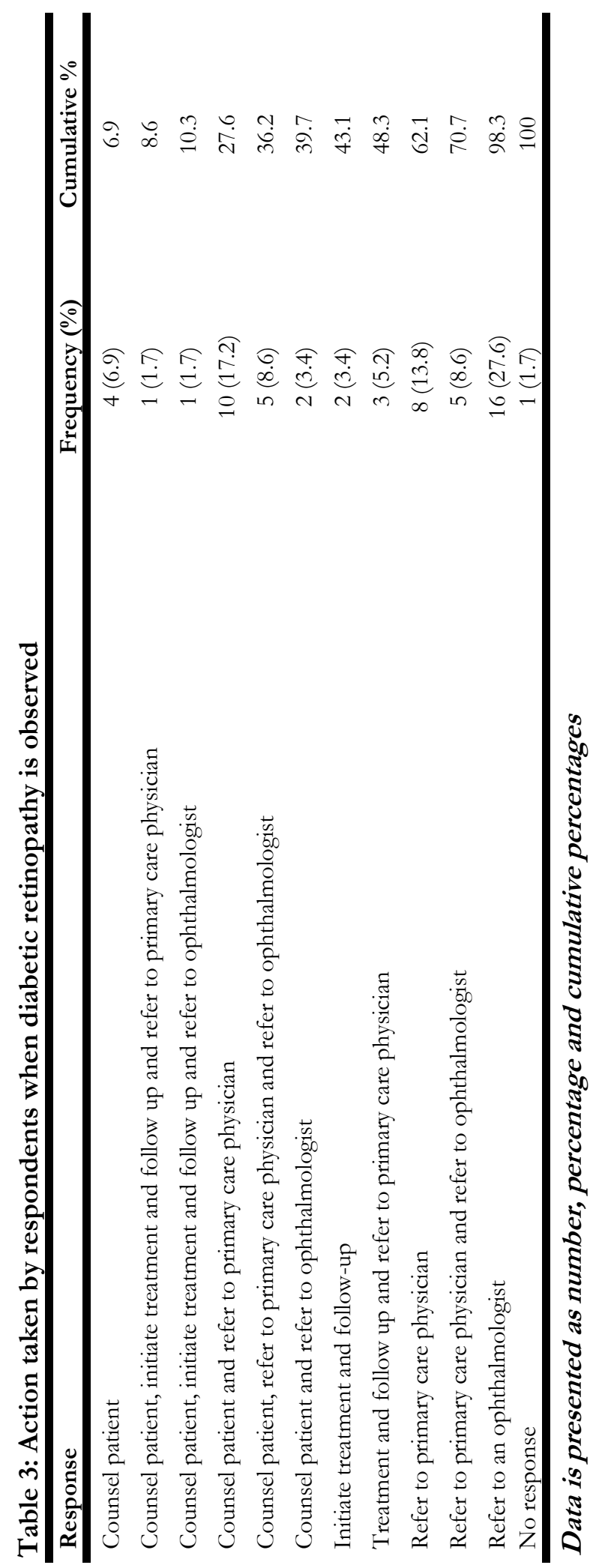




\section{Referral pattern of Optometrists in Nigeria \\ Ovenseri-Ogbomo et al.,}

will refer the patient to a medical personnel/ physician (Table 3).

With respect to the stage of diabetic retinopathy that will warrant a referral, $42(72.4 \%)$ of the respondents refer the patient at any stage when the diagnosis is made while $5(8.6 \%)$ will only refer the patient if there is no improvement in the condition presumably after follow-up care. Seven (12.1\%) and $4(6.9 \%)$ referred patients at the pre-proliferative and proliferative stage of diabetic retinopathy respectively (Table 4).

Females were more likely to refer diabetic retinopathy patients than males $(\mathrm{OR}=3.682,95 \% \mathrm{CI}=$ 0.357 - 37.922). Similarly, optometrists trained in Abia State University were more likely to refer diabetic retinopathy patients than those trained in University of Benin $(\mathrm{OR}=1.722,95 \% \mathrm{CI}=0.223$ 13.301). A further analysis of the interaction of gender with referral pattern showed that both males $(\mathrm{OR}=3.451)$ and females $(\mathrm{OR}=8.591)$ are more likely to refer patients with diabetic retinopathy than patients diagnosed with glaucoma.
Nigeria is the concern that some optometrists fail to refer patients needing ophthalmologic consult or do so belatedly. Perhaps primary open angle glaucoma which portends dire consequences when early intervention is not initiated is one of such conditions often blamed on optometrists. To provide empirical evidence to support or refute this allegation, we set out to investigate the referral behavior of Nigerian optometrists with respect to glaucoma and diabetic retinopathy.

More than $70 \%$ of respondents claim to refer glaucoma patients to an ophthalmologist with the majority of them initiating treatment before referral while $28 \%$ continue to manage the patients in their facility. We could not determine if these $28 \%$ do so independently or in co-management with ophthalmologists.

Increasingly, the concern to address the burden of blindness from glaucoma has necessitated the call for non-medical healthcare professionals to play an active role in glaucoma management (Tatham and Murdoch, 2012). Optometrists are well suited to

Table 4: Patterns of diabetic referral with respect to geo-political zones

\begin{tabular}{lcc} 
& \multicolumn{2}{c}{ Refer diabetic retinopathy patients } \\
\cline { 2 - 3 } Zone & Yes (\%) & No $(\%)$ \\
\hline South-West & $10(20.0)$ & $0(0.0)$ \\
South-East & $12(24.0)$ & $1(25.0)$ \\
South-South & $24(48.0)$ & $2(50.0)$ \\
North-Central & $3(6.0)$ & $1(25.0)$ \\
North-West & $1(2.0)$ & $0(0.0)$ \\
\hline
\end{tabular}

Data is presented as number and percentages

\section{DISCUSSION}

More often than not primary care providers refer patients to other health care providers when further care is indicated. This system of referral is intended to afford the patient the best care possible. Optometrists as primary eye care providers and given the limitation imposed on their scope of practice are statutorily under obligation to refer patients to the ophthalmologists or other healthcare providers when such referral is warranted. A common point of conflict between optometry and ophthalmology in play this role since they possess the fundamental skills required to examine glaucoma patients (Marks et al., 2012). Studies (Abrams et al., 1994; Banes et al., 2006; Marks et al., 2012) have shown a considerable agreement between optometrists and ophthalmologists in assessment of glaucoma patients. Notwithstanding this evidence, a prompt referral system of glaucoma patients for ophthalmologic consult is suggested as the patient may require an initial surgical intervention. To this end it is believed that good communication between the referring optometrists and the ophthalmologists would enhance the 
referral system. Thus, Classe and Alexander (1993) had proposed a protocol for co-management of certain conditions including cataract. They believed that since schemes of co-management exist, having a written protocol will facilitate communication between optometrists and ophthalmologists and ultimately enhance patient care.

Less than half of respondents in the South-East geopolitical zone refer glaucoma patients. There is also the finding that respondents trained in Abia State University (in the South-East zone) are less likely to refer glaucoma patients. From these two findings, we can only infer that perhaps it has to do with the curriculum. It is therefore necessary to emphasize the need for prompt referral in the curriculum of optometry training.

Studies have shown that referral for glaucoma was the second most common reason for optometrists' referral to ophthalmologists (Brin and Griffin, 1995; Weed, 1997; Pierscionek et al., 2009; Davey et al., 2011). Weed (1997) further revealed that in those referred for glaucoma, 68\% were actually diagnosed of glaucoma or were scheduled for a follow-up assessment in order to ascertain the diagnosis of glaucoma by the ophthalmologists. Furthermore, there was more concurrence of referral reason with the clinical diagnosis made by optometrists compared to general practitioners (Pierscionek et al., 2009). In the present study, $87.9 \%$ of respondents base their diagnosis of glaucoma on the triad of raised IOP, C/D ratio and characteristic visual field defect. This has a tendency to improve on the validity of the referrals thus reducing referral of false positive patients. This is critical because it has been reported that despite more concurrence between optometrists' and ophthalmologists' diagnosis of glaucoma relative to general practitioners' diagnosis, optometrists in the UK referred more false positive patients for glaucoma than did general practitioners (Pierscionek et al., 2009).

Although $44.8 \%$ of respondents use Schiotz tonometer to assess IOP, the tendency to misclassify patients based on IOP reading obtained using the Schiotz tonometer can be obviated by the fact that IOP

\section{Referral pattern of Optometrists in Nigeria}

Ovenseri-Ogbomo et al.,

is not the single criterion for diagnosing glaucoma amongst the respondents. This is further supported by the fact that $75.9 \%$ of the respondent performs VFT for their patients.

Given the irreversible damage caused by glaucoma, the need for surgical intervention when warranted and the potential for sight loss when appropriate care is delayed, it is imperative that all glaucoma patients receives ophthalmologic consult to determine the best course of management.

Diabetic retinopathy is one of the principle causes of blindness and visual impairment in Nigeria (Abdull et al., 2009). The incidence of vision loss or blindness due to ocular complications of diabetes raises sufficient public health concern, with diabetic retinopathy alone responsible for 12,000 to 24,000 new cases of blindness yearly in the United States (CDC, 2011). Early detection and prompt management are central to dealing with visual impairment due to diabetic retinopathy. At the nexus of this is appropriate and efficient referral of patients with diabetes and early signs of ocular manifestation of diabetes. In the present study, all respondents but four will refer patients when there are signs suggestive of diabetic retinopathy. Majority of the respondents would refer the patients at any stage at which a diagnosis is made. Given that management of diabetic retinopathy falls outside the scope of practice of optometrists in Nigeria, it is advocated that all patients presenting with diabetes and diabetic retinopathy be promptly referred for ophthalmologic assessment and prompt treatment.

That the odds favored the referral of diabetic retinopathy than glaucoma may relate to the fact that while some optometrists may initiate management of glaucoma, this is not the case with diabetic retinopathy. Diabetic retinopathy may present more challenge in making a diagnosis. Management of diabetic eye disease also requires ongoing diabetic control. This may account for the reason why some $13.8 \%$ of respondents will refer patients presenting with signs suggestive of diabetes to medical personnel/physicians including another $17.2 \%$ who will do the same after counseling the patients on the 


\section{Referral pattern of Optometrists in Nigeria \\ Ovenseri-Ogbomo et al.,}

cause and course of the disease. Almost all respondents indicated that they provide patient education on the cause and course of diabetic retinopathy. This is important because it has been reported that knowledge of ocular complications from diabetes mellitus is low among diabetic patients (OvenseriOgbomo et al., 2013).

\section{CONCLUSION}

Majority of Nigerian optometrists refer patients diagnosed of glaucoma and diabetic retinopathy patients to other care givers mainly ophthalmologists. Both male and female respondents were more likely to refer diabetic retinopathy than glaucoma cases with majority being female optometrists. Again, majority will initiate treatment before referral whiles a few optometrists continue to manage the patients in their facility. However, this study could not establish whether the few optometrists do so self-reliantly or in co-management with ophthalmologists.

\section{COMPETING INTERESTS}

The authors declare that they have no competing interests.

\section{REFERENCES}

Abdull M.M., Sivasubramaniam S., Murthy G.V., Gilbert C., Abubakar T., Ezelum C. and Rabiu M.M. (2009) Causes of blindness and visual impairment in Nigeria: the Nigeria national blindness and visual impairment survey. Investigative ophthalmology \& visual science 50(9), 4114-4120.

Abrams L.S., Scott I.U., Spaeth G.L., Quigley H.A. and Varma R. (1994) Agreement among optometrists, ophthalmologists, and residents in evaluating the optic disc for glaucoma. Ophthalmology 101(10), 1662-1667.

Banes M., Culham L., Bunce C., Xing W., Viswanathan A. and Garway-Heath D. (2006) Agreement between optometrists and ophthalmologists on clinical management decisions for patients with glaucoma. British Journal of Ophthalmology 90(5), 579-585.

Bowling B., Chen S. and Salmon J. (2005) Outcomes of referrals by community optometrists to a hospital glaucoma service. British Journal of Ophthalmology 89(9), 1102-1104.

Brin B.N. and Griffin J.R. (1995) Referrals by optometrists to ophtholmologists and other providers.

CDC (2011) Centers for Disease Control Prevention, National diabetes fact sheet: national estimates and general information on diabetes and prediabetes in the United States, 2011. In Atlanta, GA: US Department of Health and Human Services, Centers for Disease Control and Prevention.

Classe J. and Alexander L. (1993) Protocols for comanagement. Optometry clinics: the official publication of the Prentice Society 4(2), 101-122.

Davey C.J., Green C. and Elliott D.B. (2011) Assessment of referrals to the hospital eye service by optometrists and GPs in Bradford and Airedale. Ophthalmic and Physiological Optics 31(1), 23-28.

Harrison R., Wild J. and Hobley A. (1988) Referral patterns to an ophthalmic outpatient clinic by general practitioners and ophthalmic opticians and the role of these professionals in screening for ocular disease. Bmj 297 (6657), 1162-1167.

Marks J., Harding A., Harper R., Williams E., Haque S., Spencer A. and Fenerty C. (2012) Agreement between specially trained and accredited optometrists and glaucoma specialist consultant ophthalmologists in their management of glaucoma patients. Eye 26(6), 853-861.

Ovenseri-Ogbomo G.O., Abokyi S., Koffuor G. and Abokyi E. (2013) Knowledge of diabetes and its associated ocular manifestations by diabetic patients: A study at Korle-Bu Teaching Hospital, Ghana. Nigerian medical journal: journal of the Nigeria Medical Association 54(4), 217.

Pierscionek T., Moore J. and Pierscionek B. (2009) Referrals to ophthalmology: optometric and general practice comparison. Ophthalmic and Physiological Optics 29(1), 32-40.

Tatham A. and Murdoch I. (2012) The effect of appointment rescheduling on monitoring 


\section{Referral pattern of Optometrists in Nigeria}

Ovenseri-Ogbomo et al.,

interval and patient attendance in the glaucoma outpatient clinic. Eye 26(5), 729-733.

WCO (2014) World Council of Optometry: Who is an optometrist? [Online].
Weed K.H. (1997) Optometric referrals to an ophthalmology outpatients department: classification and sensitivity. Ophthalmic and Physiological Optics 17(6), 541.
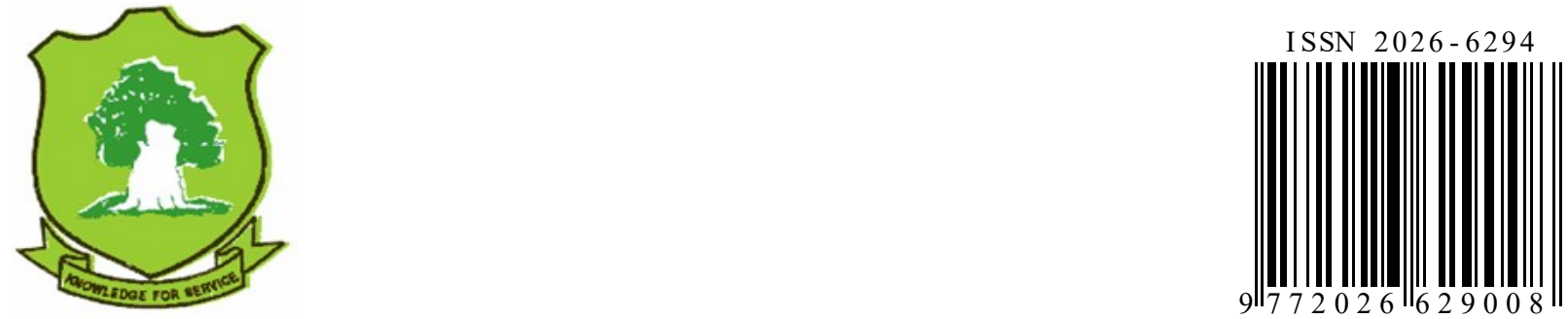